\title{
Current Status and Future Plan of Osaka Prefecture University 1.85-m mm-submm Telescope Project
}

\author{
Atsushi Nishimura ${ }^{a}$, Kazuki Tokuda ${ }^{a}$, Ryohei Harada ${ }^{\mathrm{a}}$, Yutaka Hasegawa ${ }^{\mathrm{a}}$, Shota Ueda ${ }^{\mathrm{a}}$, Sho \\ Masui $^{\mathrm{a}}$, Ryotaro Konishi ${ }^{\mathrm{a}}$, Yasumasa Yamasaki ${ }^{\mathrm{a}}$, Hiroshi Kondo ${ }^{\mathrm{a}}$, Koki Yokoyama ${ }^{\mathrm{a}}$, Takeru \\ Matsumoto $^{\mathrm{a}}$, Taisei Minami ${ }^{\mathrm{a}}$, Masanari Okawa ${ }^{\mathrm{a}}$, Shinji Fujita ${ }^{\mathrm{a}}$, Ayu Konishia ${ }^{\mathrm{a}}$, Yuka Nakao ${ }^{\mathrm{a}}$, \\ Shimpei Nishimoto ${ }^{a}$, Sana Kawashita ${ }^{a}$, Sho Yoneyama ${ }^{a}$, Tatsuyuki Takashima ${ }^{a}$, Kenta Goto ${ }^{a}$, \\ Nozomi Okada $^{a}$, Kimihiro Kimura ${ }^{a}$, Yasuhiro Abe ${ }^{a}$, Kazuyuki Muraoka ${ }^{a}$, Hiroyuki Maezawa ${ }^{a}$, \\ Toshikazu Onishi ${ }^{a}$, and Hideo Ogawa ${ }^{a}$ \\ aDepartment of Physical Science, Graduate School of Science, Osaka Prefecture University, 1-1 \\ Gakuen-cho, Naka-ku, Sakai, Osaka 599-8531, Japan
}

\begin{abstract}
We report the current status of the 1.85-m mm-submm telescope installed at the Nobeyama Radio Observatory (altitude $1400 \mathrm{~m}$ ) and the future plan. The scientific goal is to reveal the physical/chemical properties of molecular clouds in the Galaxy by obtaining large-scale distributions of molecular gas with an angular resolution of several arcminutes. A semi-automatic observation system created mainly in Python on Linux-PCs enables effective operations. A large-scale CO $J=2-1$ survey of the molecular clouds (e.g., Orion-A/B, Cygnus-X/OB7, TaurusCalifornia-Perseus complex, and Galactic Plane), and a pilot survey of emission lines from minor molecular species toward Orion clouds have been conducted so far. The telescope also is providing the opportunities for technical demonstrations of new devices and ideas. For example, the practical realizations of PLM (Path Length Modulator) and waveguide-based sideband separating filter, installation of the newly designed waveguide-based circular polarizer and OMT (Orthomode Transducer), and so on. As the next step, we are now planning to relocate the telescope to San Pedro de Atacama in Chile (altitude $2500 \mathrm{~m}$ ), and are developing very wideband receiver covering 210-375 GHz (corresponding to Bands 6-7 of ALMA) and full-automatic observation system. The new telescope system will provide large-scale data in the spatial and frequency domain of molecular clouds of Galactic plane and Large/Small Magellanic Clouds at the southern hemisphere. The data will be precious for the comparison with those of extra-galactic ones that will be obtained with ALMA as the Bands 6/7 are the most efficient frequency bands for the surveys in extra-galaxies for ALMA.
\end{abstract}

Keywords: Osaka 1.85-m telescope, radio telescope, winde band receiver, telescope control system, ROS, NECST, Atacama

\section{INTRODUCTION}

The rotational transition line of $\mathrm{CO}$ is one of the most important emission line in astrophysics to investigate the distribution, physical properties, and kinematics of the interstellar medium (ISM). Especially, the lines of CO $J=1-0$ and its isotopes have been widely used as a probe of the mass of molecular contents of the ISM, because of its high abundance, low critical density for excitation, and relatively stable chemical properties. The distribution of the line in the Galaxy has been explored with relatively small-aperture telescopes, such as the 1.2-m telescopes at the Harvard-Smithsonian Center for Astrophysics (CfA) and Cerro Tololo Inter-American Observatory in Chile, ${ }^{1-3}$ as well as the 4-m telescope in Nagoya and the NANTEN telescope in Chile. ${ }^{4-7}$

Although the $J=1-0$ lines of $\mathrm{CO}$ are powerful tools to investigate the mass of the molecular cloud, the other transitions with different critical densities for the excitation are needed to investigate the local density and the temperature of the ISM. However, such observations targeting the higher transition lines of CO were conduced only at coarse angular resolutions such as a survey of $60-\mathrm{cm}$ telescope ${ }^{8-11}$ or only toward small regions such as a

Further author information: (Send correspondence to A.N.)

A.N.: E-mail: nishimura@p.s.osakafu-u.ac.jp 
survey of JCMT/HARP, ${ }^{12,13}$ at the time of early 2010s, mainly because the development of sensitive receivers at high frequencies has been very difficult and also because the opacity of the Earth's atmosphere is high at low altitude site.

The 1.85-m mm-submm telescope was developed to realize a large-scale CO $J=2-1$ survey. The first science operation was started on November 2011 by using a sideband-separating (2SB) SIS receiver, and the CO survey was continued several years. The developments of receiver was continued during the operation, and the $1.85-\mathrm{m}$ telescope was also used as a test bench of the new receiver concepts. In this paper, we describe the history of the developments and observations of the 1.85-m telescope, current status of the project and the future plan in detail.

\section{DEVELOPMENT OF RADIO INSTRUMENTS}

The 1.85-m telescope ${ }^{14}$ has a Cassegrain reflector antenna with Nasmyth beam-waveguide feed, and is mounted on an azimuth-elevation rotating structure (Fig 1). The main reflector is made of carving of one piese of aluminium, and its surface accuracy was measured to be $19 \mu \mathrm{m}$ by a 3-D coordinate measuring machine, SNK MM-3500. The radome is equipped to protect the telescope from wind, precipitation, and sunlight, that was originally used for the 1.2-m telescope installed at the summit of Mt. Fuji. ${ }^{15}$ The path length modulator ${ }^{16}$ (PLM) was installed in front of the RF window of the receiver to reduce standing waves between the RF window and the calibration unit or the $\mathrm{Al}$ frames of the radome. For more detail on the antenna and optics, see Ref. 14.

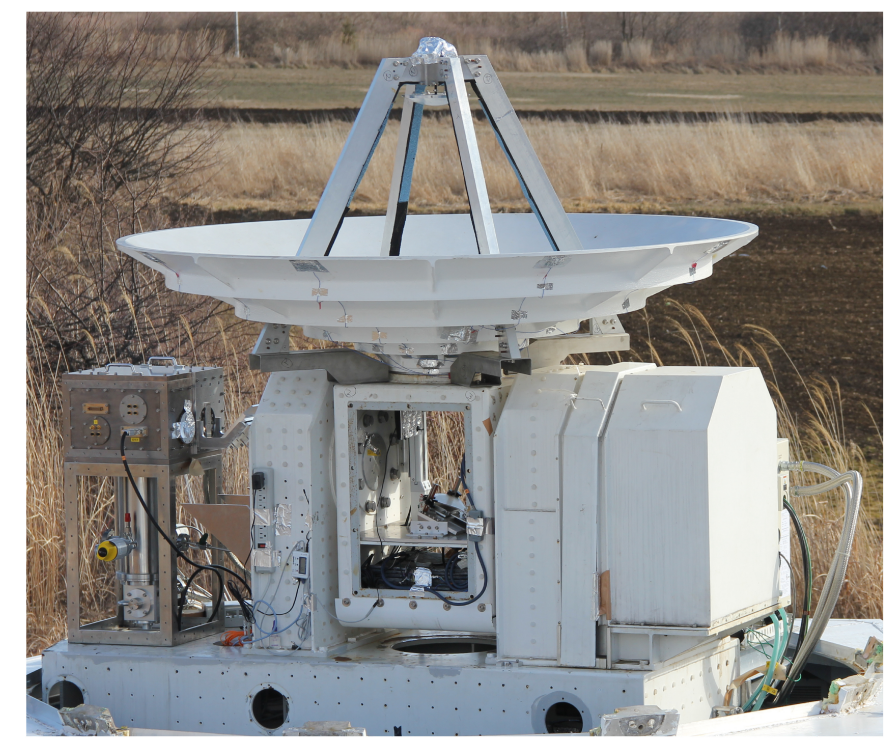

Figure 1. Photograph of the $1.85-\mathrm{m}$ telescope at the Nobeyama Radio Observatory.

The construction of the telescope at the Nobeyama Radio Observatory (NRO) was started on 2007, and the first simultaneous observations of the ${ }^{12} \mathrm{CO},{ }^{13} \mathrm{CO}$, and $\mathrm{C}^{18} \mathrm{O}$ of $J=2-1$ transition lines were achieved in September 2009. The beam size was measured to be $2.7^{\prime}$ at $230 \mathrm{GHz}$ band. Fig. 2 summarizes the receiver system and control system developed and used for the 1.85-m telescope.

\subsection{Receiver System}

\subsubsection{Season 2009-2012}

The first light receiver for the 1.85-m telescope was a balanced-type sideband-separating (2SB) SIS receiver. The basic design is the same as Ref. 17 which was developed for the 60-cm AMANOGAWA telescope. The target LO frequency is $\sim 225 \mathrm{GHz}$, and the receiver outputs two IF signals with a frequency of 4-8 GHz. In order to achieve simultaneous observations of ${ }^{12} \mathrm{CO} J=2-1(230.538 \mathrm{GHz}),{ }^{13} \mathrm{CO} J=2-1(220.399 \mathrm{GHz})$, and $\mathrm{C}^{18} \mathrm{O}$ 


\begin{tabular}{|c|c|c|c|c|}
\hline FY & Polarizer & $\begin{array}{c}\text { Sideband } \\
\text { Separation }\end{array}$ & Spectrometer & Remote Control \\
\hline 2010 & \multirow{2}{*}{ None } & \multirow{3}{*}{$\begin{array}{l}\text { Balanced } \\
\text { 2SB-Mixer }\end{array}$} & \multirow{4}{*}{ Acqiris AC240 } & \multirow{8}{*}{$\begin{array}{l}\text { C / Python based socket } \\
\text { control system }\end{array}$} \\
\hline 2011 & & & & \\
\hline 2012 & \multirow{2}{*}{ OMT } & & & \\
\hline 2013 & & \multirow{3}{*}{$\begin{array}{l}\text { 220/230 GHz } \\
\text { 2SB-Filter }\end{array}$} & & \\
\hline 2014 & \multirow{6}{*}{ SST-CP } & & \multirow{6}{*}{ RPG XFFTS } & \\
\hline 2015 & & & & \\
\hline 2016 & & \multirow{4}{*}{$\begin{array}{c}220 / 230 \mathrm{GHz} \\
+230 / 240 \mathrm{GHz} \\
\text { 2SB-Filter }\end{array}$} & & \\
\hline 2017 & & & & \\
\hline 2018 & & & & Python / ROS based \\
\hline 2019 & & & & $\begin{array}{l}\text { contro1 system } \\
\text { (NECST) }\end{array}$ \\
\hline
\end{tabular}

Figure 2. History of the installed receiver and control system of the 1.85-m telescope.

$J=2-1(219.560 \mathrm{GHz})$ by using one spectrometer with a bandwidth of $1 \mathrm{GHz}$, Acqiris AC240, ${ }^{18}$ two output signals of the SIS receiver (1st IF) were split to 3 signals, then each of the signal was further down-converted to different frequency in the 2 nd IF band, and finally those 3 signals merged and one $0-1 \mathrm{GHz}$ signal which contains the 3 different RF bands was generated in the IF system. ${ }^{14}$

In the observation season of 2012, an Orthomode Transducer ${ }^{19,20}$ (OMT) for dual-polarization observations was developed and installed to the telescope. The measured characteristics of the OMT were an insertion loss of less than $0.5 \mathrm{~dB}$, return loss of better than $20 \mathrm{~dB}$, and cross polarization coupling of better than $20 \mathrm{~dB}$ across 210-250 GHz. ${ }^{14}$ Using the OMT with two balanced-type 2SB SIS receivers, observation efficiency was improved by a factor of 2 than previous seasons.

\subsubsection{Season 2013-2015}

Although the balanced-type 2SB SIS mixer receivers are very powerful to observe wide frequency ranges simultaneously, its image rejection ratio (IRR) is relatively low, ${ }^{21,22}$ typically $10 \mathrm{~dB}$, and the stability of the IRR is not

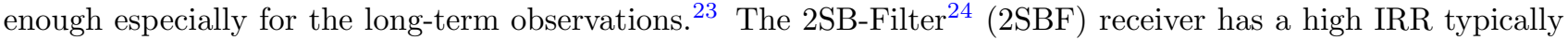
more than $20 \mathrm{~dB}$, and the long-term stability. In the $2 \mathrm{SBF}$ receiver, the cutout frequency of the lower sideband (LSB) and upper sideband (USB) are determined at the design of the filter implemented in waveguide. The target emission lines of the 1.85-m telescope was CO $J=2-1$, and other lines were not planned to observe the season, thus it was not a problem even if the LO frequency was fixed. We developed a 2SBF receiver optimized for ${ }^{12} \mathrm{CO},{ }^{13} \mathrm{CO}$, and $\mathrm{C}^{18} \mathrm{O} J=2-1$ lines observations. ${ }^{25}$ The IRR of the receiver was measured to be better than $25 \mathrm{~dB}$.

The spectrometer was replaced on the season of 2013 with a bandwidth of $2 \mathrm{GHz}$ model, RPG XFFTS. ${ }^{26}$ We installed four XFFTS boards, and use each board for each receiver output: one board for the ${ }^{12} \mathrm{CO}$ band and another board for the ${ }^{13} \mathrm{CO}$ and $\mathrm{C}^{18} \mathrm{O}$ band.

In the observation season of 2014, we replaced the OMT to a stepped septum-type waveguide circular polar$\operatorname{izer}^{27}$ (SST-CP). By this replacement, the 1.85-m telescope was ready to use as a base of VLBI observations.

\subsubsection{Season 2016-2019}

By the season of 2015, the survey observations of CO $J=2-1$ was almost completed for the Galactic plane on the northern hemisphere (see also Section 3.1), so we changed the science goal to survey weaker emission line in the $220 \mathrm{GHz}$ band such as $\mathrm{SO}, \mathrm{CS}$, and $\mathrm{CH}_{3} \mathrm{OH}$. In order to achieve the new theme, we developed the new filter system based on 2SBF (220/230/240 Rx). In the system, the polarization of the received radio wave was firstly separated by the SST-CP, then signals of each of polarization are derived to the two 2SBFs which were tuned for the different RF frequency (Fig. 3): one for 220 and $230 \mathrm{GHz}$, and another for 230 and $240 \mathrm{GHz}$. The typical system noise temperature $\left(T_{\text {sys }}\right)$ of the $220 / 230 / 240 \mathrm{Rx}$ was measured to be $80,90,100$, and $150 \mathrm{~K}$ for 220, 230 (LHCP), 230 (RHCP), and $240 \mathrm{GHz}$ band, respectively, measured with the 1.85-m telescope at NRO. 

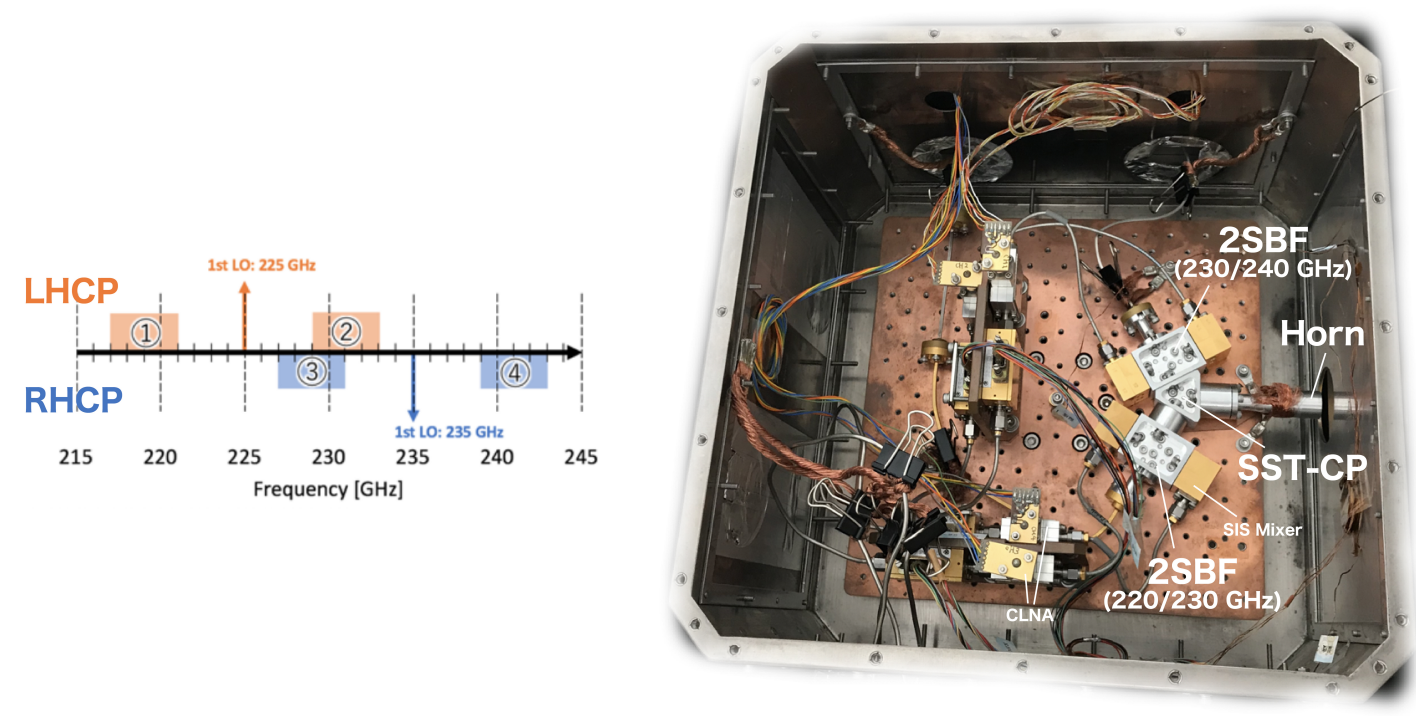

Figure 3. (Left) Schematic diagram of the frequency separation in the 220/230/240 receiver. (Right) Photograph of the $220 / 230 / 240$ receiver.

\subsection{Telescope Control System}

The telescope and various equipment including the spectrometers are controlled and monitored on a Linux PC system via TCP/UDP/IP socket connections. The whole system consists of the following components: 1, Hardware driver layer consisting of programs for controlling each of the instrument (e.g., telescope drives, encoders, receiver equipment); 2, Communication layer consisting of programs which command the instruments in cooperation to realize the observations; 3, Database layer consisting of programs that collect and store the data including the spectrometer output, environmental parameters, and operation log.

\subsubsection{Season 2009-2017}

The control system for the 1.85-m telescope was started with a following composition. The hardware driver layer was mostly written in C language to control the PCI boards, and written as a TCP server. The communication layer was written in Python, and the database was implemented integrally on MySQL database system. The detail of the implementation of control system is described in Ref. 14. Based on the framework, the queue based semi-automatic operation was realized which reduced burden for the telescope operator and the time loss due to the careless mistake, and hence improved the actual observation efficiency (Fig. 4). Furthermore, we implemented the automatic data analyzing pipeline that provides observation results graphically via the web interface soon after the finishing each observation (Fig. 5).

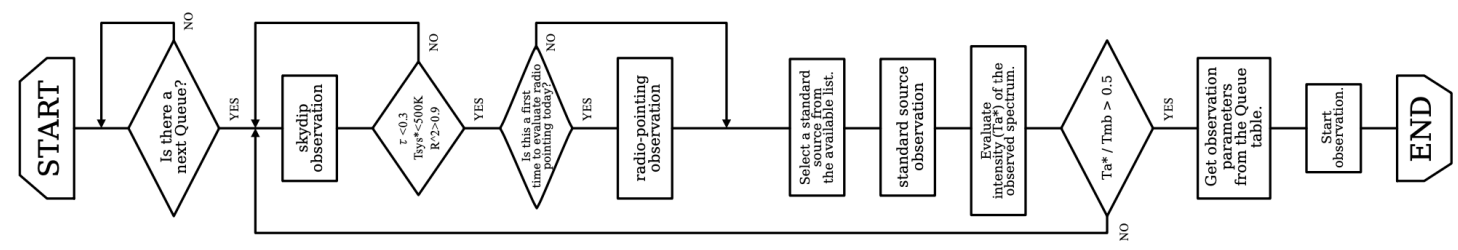

Figure 4. Flowchart of the queue based semi-automatic observation implemented in the observation system on the $1.85-\mathrm{m}$ telescope. 


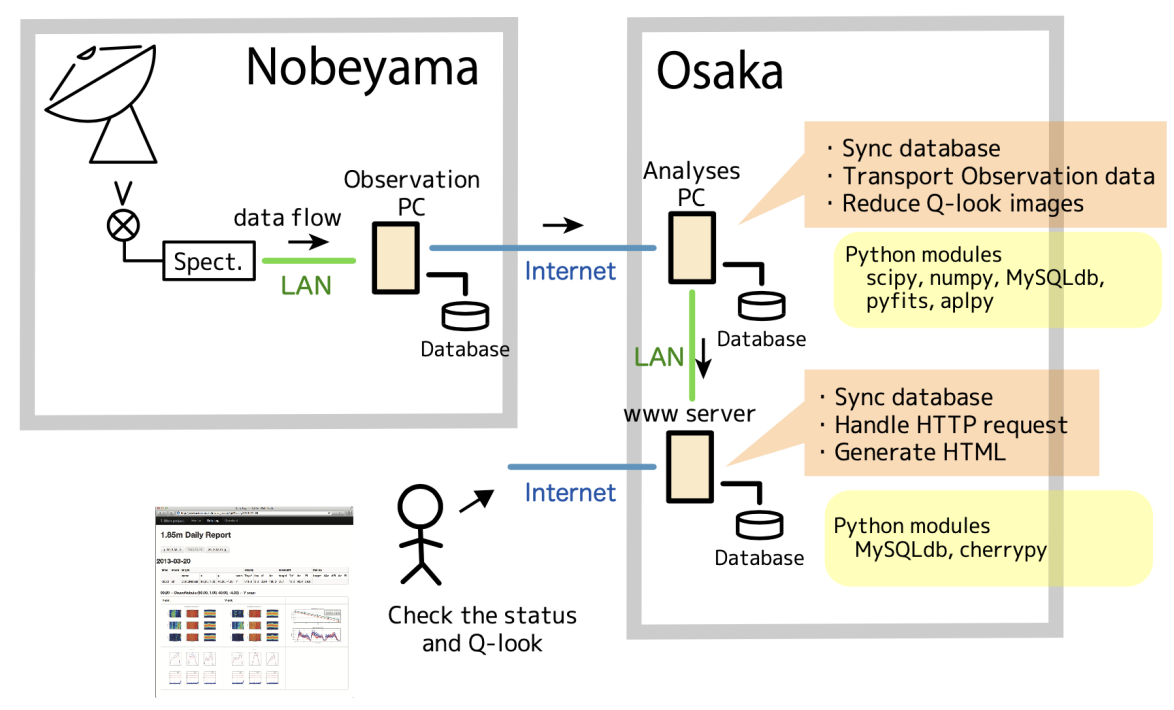

Figure 5. Schematic diagram of the web-based Q-look system.

\subsubsection{Season 2018-2019}

From the observation season of 2018, we started to develop a new control system based on ROS $^{28}$ (robot operating system). The ROS is one of the most prevailing open source framework to control robots and provides a very useful communication module (called Topic) which realizes many-to-many communications between processes and/or computers. The reliability and scalability on the communication layer was improved by introducing the Topic based system. In addition, aiming to realize further efficiency on developing time, we developed the fully python based hardware drivers, pypci* and pyinterface ${ }^{\dagger}$. The detail of the ROS based control system NECST is described in Ref. 29, and the performance evaluations of the new control system is ongoing.

*https://github.com/ars096/pypci

${ }^{\dagger}$ https://github.com/ogawa-ros/pyinterface

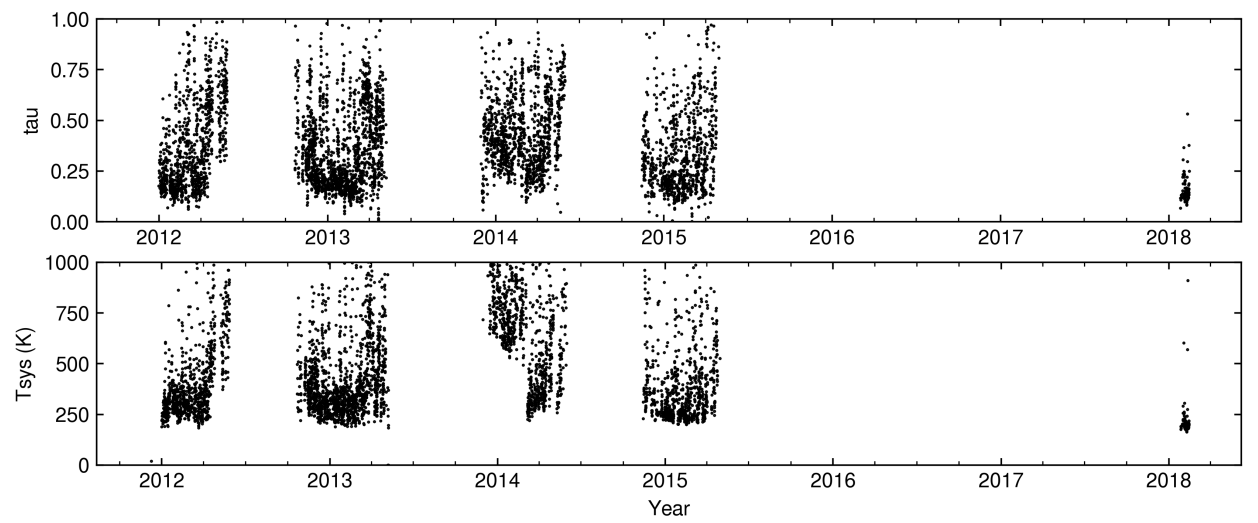

Figure 6. Distribution of the optical depth (upper) and the system noise temperature $\left(T_{\text {sys }}\right)$ including the atmosphere toward the zenith (lower) measured by the 1.85-m telescope with a frequency band of $230 \mathrm{GHz}$. Before 2012, the integrated database system was not installed, so the environmental data before that time was lost. 


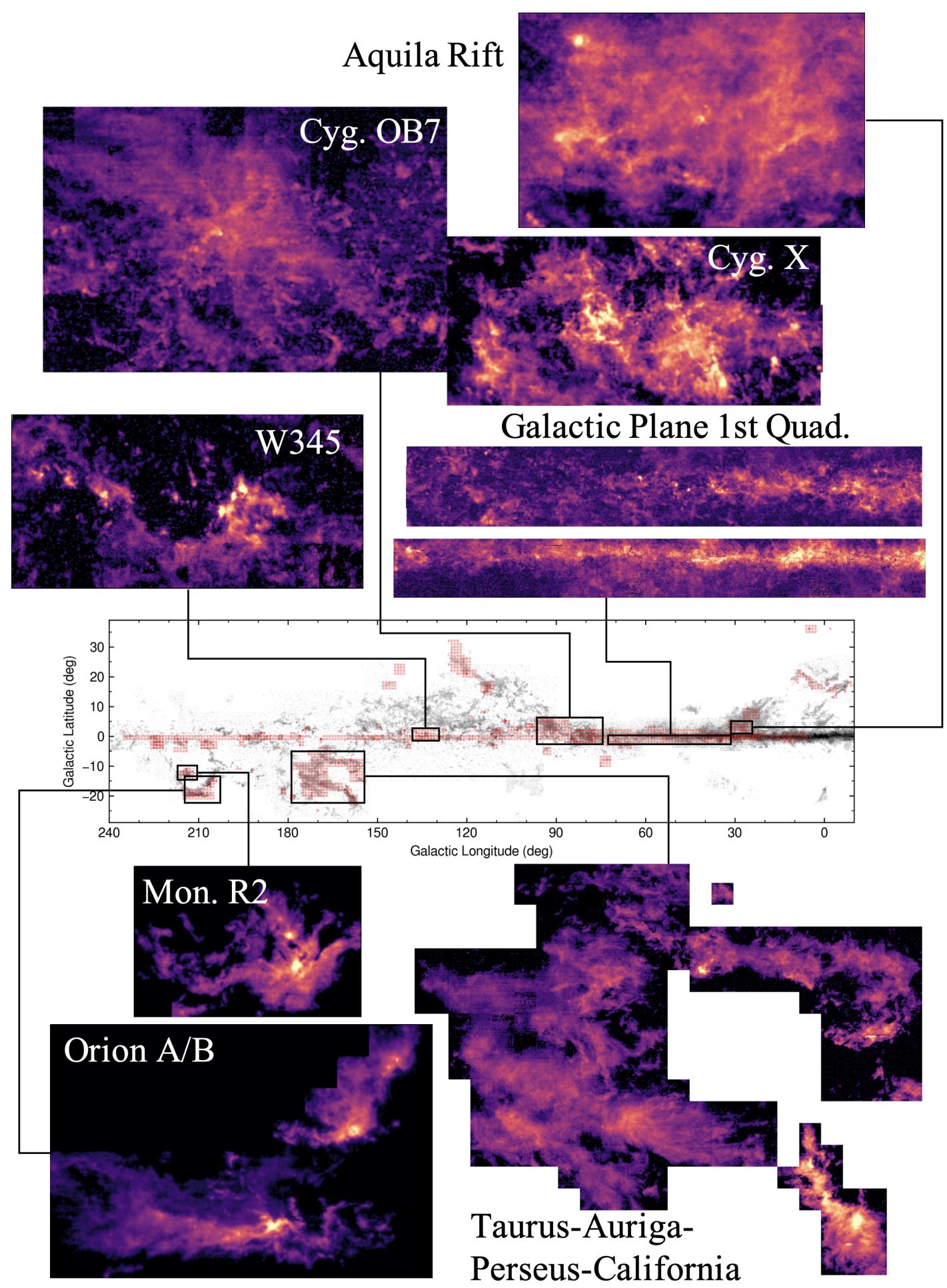

Figure 7. Middle: Observation coverage of the $1.85-\mathrm{m}$ CO $J=2-1$ survey. Background gray-scale image shows the distribution of ${ }^{12} \mathrm{CO} J=1-0$ taken by Ref. 3 as reference. Red squares indicate observed area by the $1.85-\mathrm{m}$ telescope. Upper and lower: Some samples of the distributions of CO $J=2-1$ toward the famous star formation regions and the Galactic plane. Color ranges are different each other. 


\section{OBSERVATIONS}

The science operation was started on 2011. Usually, the science observations were conducted during the winter season (from November to next April), and the telescope was shut-down during the summer season because the high humidity condition reduces observation efficiency from the ground. Figure 6 shows the distribution of the optical depth and the system noise temperature $\left(T_{\text {sys }}\right)$ including the atmosphere measured toward the zenith with a frequency band of $230 \mathrm{GHz}$ at NRO. About the $60 \%$ of the observation seasons show a better sky condition than the threshold of $T_{\text {sys }}<400 \mathrm{~K}$ and the optical depth of $<0.4$. For the seasons of 2010-2014 (Nov. 2010 to Apr. 2015), we performed the large scale survey of CO $J=2-1$, for the seasons of 2015-2016 were devoted to the demonstration experiments for the new instruments described in Section 2.1, and we conducted the observations of multiple line mapping in the season 2017 (Nov. 2017 to Apr. 2018).

\subsection{Survey Observations of CO $\mathrm{J}=2-1$ Lines}

Fig. 7 shows the survey coverage of the 1.85 -m observations and some examples of obtained CO $J=2-1$ maps. Totally $1800 \mathrm{deg}^{2}$ area (corresponding to $4.4 \%$ of all-sky) was observed in the seasons of 2010, 2011, 2012, 2013, and 2014, and the observed areas for each season were 323, 160, 729, 303, and $307 \mathrm{deg}^{2}$, respectively. All observations were conducted by the On-the-Fly (OTF) mapping mode with an angular spacing of $1^{\prime}$ grid. The number of obtained spectra is $6,480,000$. The spatial resolution after the data reduction was $\sim 3^{\prime}$, and the typical noise level was $T_{\text {rms }} \sim 0.8 \mathrm{~K}$ with a velocity resolution of $\sim 0.1 \mathrm{~km} \mathrm{~s}^{-1}$. Using the CO data, studies on the star formation, ${ }^{30-39}$ and on the $\mathrm{ISM}^{40,41}$ were promoted.

From the series of CO $J=2-1$ observations toward the GMCs in the Galaxy, we found that ${ }^{12} \mathrm{CO} J=2-$ 1 is easily becoming optically thick at the inner part of the GMC where the column density is higher than $\sim 3 \times 10^{21} \mathrm{~cm}^{-2}$, and hence the ratio of ${ }^{12} \mathrm{CO} J=2-1 /{ }^{12} \mathrm{CO} J=1-0, R_{2-1 / 1-0}^{12}$, is observed as $\sim 0.7$ which is a value expected to be observed in the condition of the local thermodynamic equilibrium (LTE) by the numerical simulations. ${ }^{42}$ On the other hand, we found that the ratio of ${ }^{13} \mathrm{CO} J=2-1 /{ }^{13} \mathrm{CO} J=1-0, R_{2-1 / 1-0}^{13}$, is a good tracer of the volume density even toward the inner region of the GMC. For the case of Orion GMCs, the observed $R_{2-1 / 1-0}^{13}$ is in the range of $0.2-2.0$, and its variation is clearly seen even toward the most inner part of the GMC where the column density is $\sim 10^{22} \mathrm{~cm}^{-2}{ }^{31}$ The volume density of $800-2000 \mathrm{~cm}^{-3}$ is estimated in the region including the inner part of the GMC by using LVG analysis.

\section{LHCP}
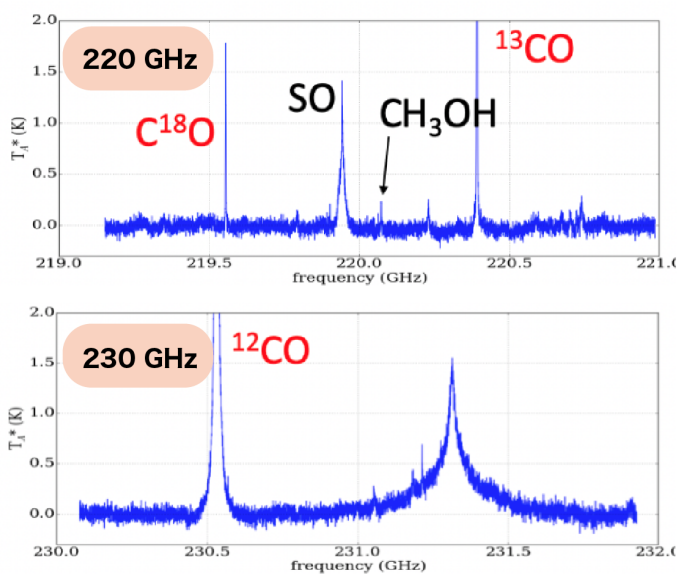
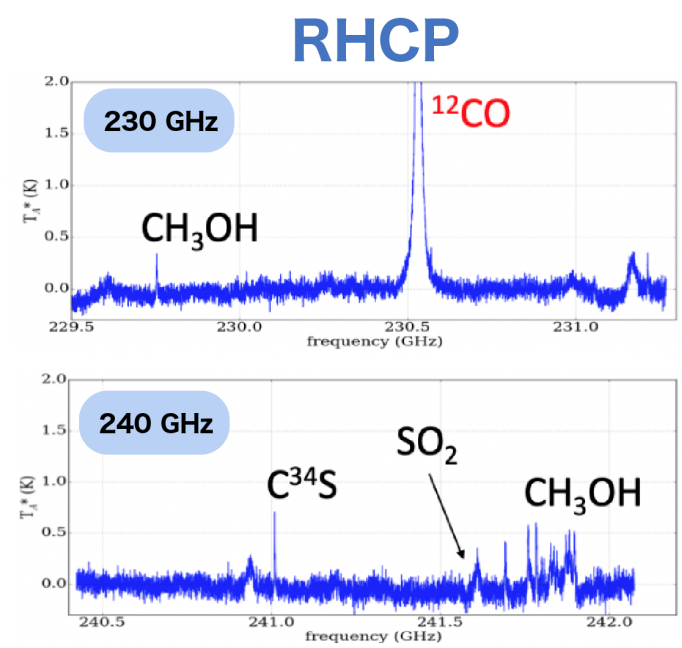

Figure 8. Spectra observed toward the Orion-KL region. 

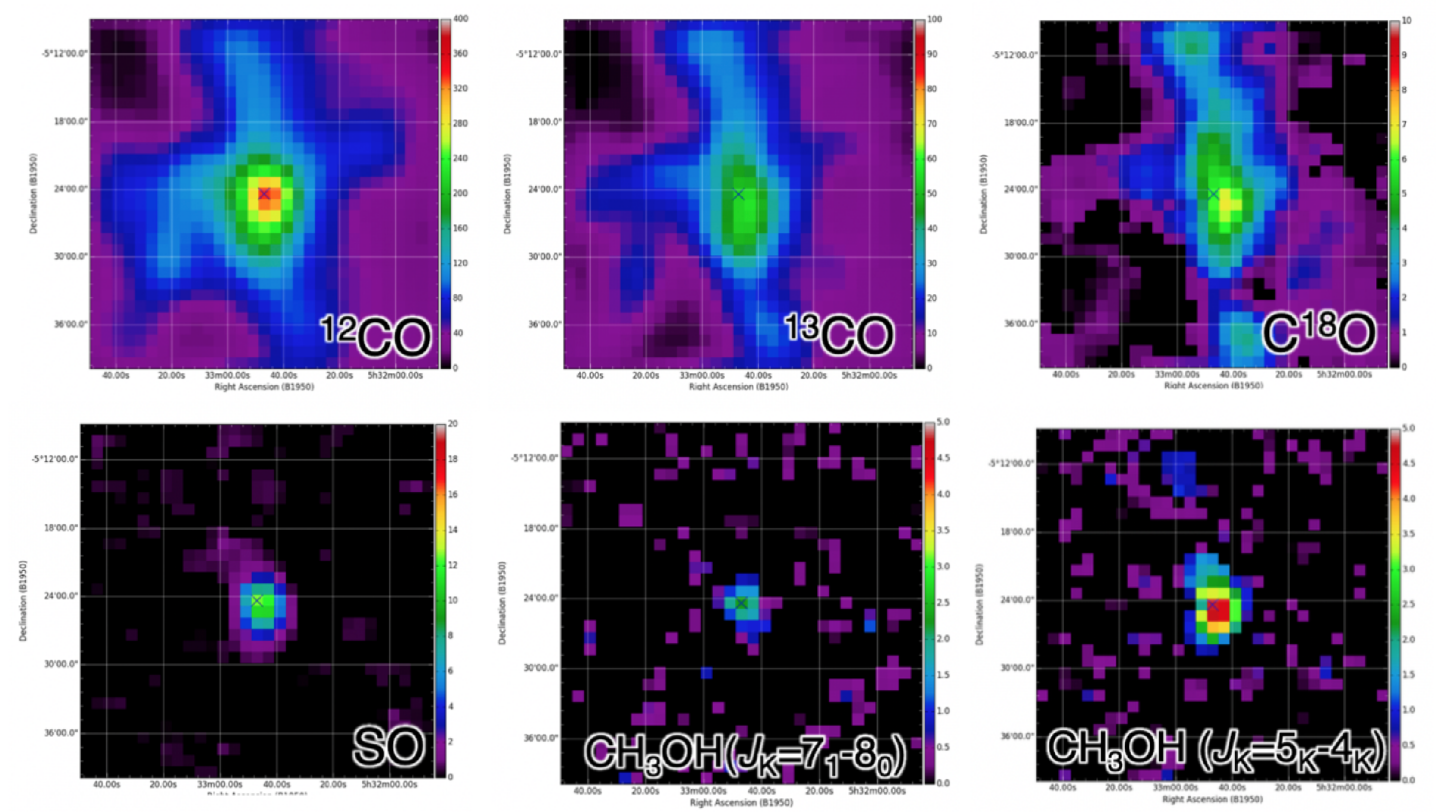

Figure 9. Maps of ${ }^{12} \mathrm{CO},{ }^{13} \mathrm{CO}, \mathrm{C}^{18} \mathrm{O}, \mathrm{SO}$, and $\mathrm{CH}_{3} \mathrm{OH}$ observed toward the Ori-KL region.

\subsection{Spectral Line Surveys for $230 \mathrm{GHz}$ Band}

In the early 2018, the test observations of the $2 \mathrm{SBF}$ receiver was conducted. The receiver has four windows for observations: $219-221 \mathrm{GHz}$ band including ${ }^{13} \mathrm{CO}, \mathrm{C}^{18} \mathrm{O}$, SO, and $\mathrm{CH}_{3} \mathrm{OH}$ lines; 229.5-231.5 $\mathrm{GHz}$ band of the RHCP side including ${ }^{12} \mathrm{CO}$ and $\mathrm{CH}_{3} \mathrm{OH}$ lines; $230-232 \mathrm{GHz}$ band of the LHCP side including ${ }^{12} \mathrm{CO}$ line; and 240.5-242.5 GHz band including $\mathrm{C}^{34} \mathrm{~S}, \mathrm{SO}_{2}$, and $\mathrm{CH}_{3} \mathrm{OH}$ lines. Fig. 8 shows the spectra observed toward the Ori-KL region. All spectra listed above were simultaneously detected. Fig. 9 shows maps of ${ }^{12} \mathrm{CO},{ }^{13} \mathrm{CO}, \mathrm{C}^{18} \mathrm{O}$, $\mathrm{SO}$, and $\mathrm{CH}_{3} \mathrm{OH}$ observed toward the Ori-KL region. These results demonstrate the capability for the wide-band observations of the $2 \mathrm{SBF}$ receiver.

\subsection{Technology Verification on VLBI Observations in $230 \mathrm{GHz}$ Band}

The experiment on VLBI observations in $230 \mathrm{GHz}$ band was conducted on April 2015 between two bases using the 1.85-m telescope and the SPART telescope which is a single dish operation mode of Nobeyama millimeter array. The length of the baseline was $150 \mathrm{~m}$, and the instruments for the VLBI observations (e.g., a frequency standard generator, samplers) were brought to the telescope site in temporarily. By observing the moon edge, VLBI fringe was successfully detected by the software correlator. This success of the experiment led to the realization of the next VLBI experiment among the SPART 10-m, SRAO 6-m in Korea, and GLT 12-m in Greenland with a frequency of $230 \mathrm{GHz}$.

\section{FUTURE PLAN}

In order to extend the observation frequency band further wide, and to achieve simultaneous observations of 230 and $345 \mathrm{GHz}$ band by sharing same single beam, ${ }^{43,44}$ we are planing to relocate the 1.85 -m telescope to San Pedro de Atacama (SPdA), Chile (alt. $2400 \mathrm{~m}$ ). Although, the altitude of SPdA is not high as the Atacama high site where NANTEN2 and ASTE are installed, its weather condition would be almost sufficient for observations of 230 and $345 \mathrm{GHz}$ band. On the contrary, SPdA would be better choice in terms of the cost of construction and running. The 1.85-m telescope will be installed in the base facility of the TAO project as part of the cooperative research with the university of Tokyo. Fig. 10 shows a photograph of the planned site of the 1.85-m 

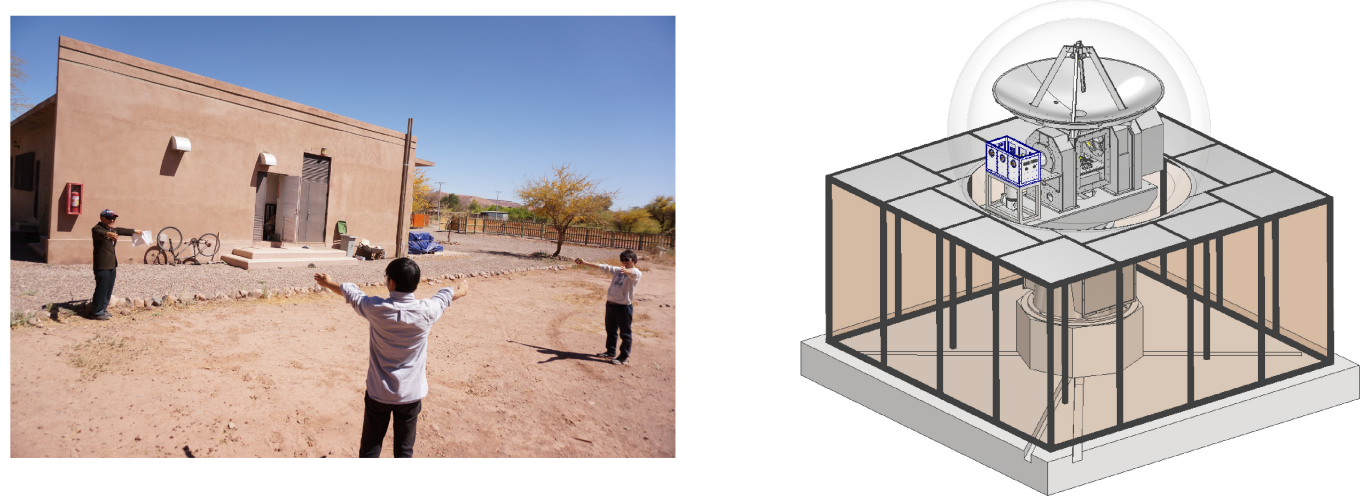

Figure 10. Left: Photograph of the planned location of the 1.85-m telescope installation in the base facility of the TAO project. Persons, Prof. Hideo Ogawa, Mr. Yasumasa Yamasaki, and Mr. Takeru Matsumoto from left to right, indicate the size of the telescope base shown in the right figure. Right: CAD drawing of the model of telescope base which will be constructed at the telescope site in SPdA.

telescope installation and a CAD drawing of the new telescope base which will be constructed to the site. By this relocation, we will be able to perform the verification tests for the higher frequency instruments and to observe the higher frequency bands, as well as to observe southern sky including the Large/Small Magellanic Clouds.

\section{SUMMARY}

In the paper, we reviewed and summarized the activities of developments and observations on the 1.85-m telescope installed at Nobeyama Radio Observatory (NRO), Japan. The construction of the telescope at NRO was started on 2007. The 2SB receiver with a $\mathrm{RF}$ of $230 \mathrm{GHz}$ was installed, and its first light was achieved on September 2009 by observing the ${ }^{12} \mathrm{CO},{ }^{13} \mathrm{CO}$, and $\mathrm{C}^{18} \mathrm{O} J=2-1$ lines simultaneously. The science observation operations were started on November 2011, and the large-scale mapping observations of the ${ }^{12} \mathrm{CO},{ }^{13} \mathrm{CO}$, and $\mathrm{C}^{18} \mathrm{O} J=2-1$ lines were continued to the observation season of 2014. Finally, an area of $1800 \mathrm{deg}^{2}$ was observed and a number of 6,480,000 spectra was obtained. During the period of science operations, the developments of the receivers and control systems were also continued. Those developed systems were installed to the telescope in the summer season, when the science observations were stopped, and were tested for verification, and then, were used for scientific observations. To date, 18 of refereed journal papers, 3 of doctor theses, 23 of master theses, and 31 of graduation theses were published related to the telescope or by using the data obtained as the CO survey (Fig. $11)$.

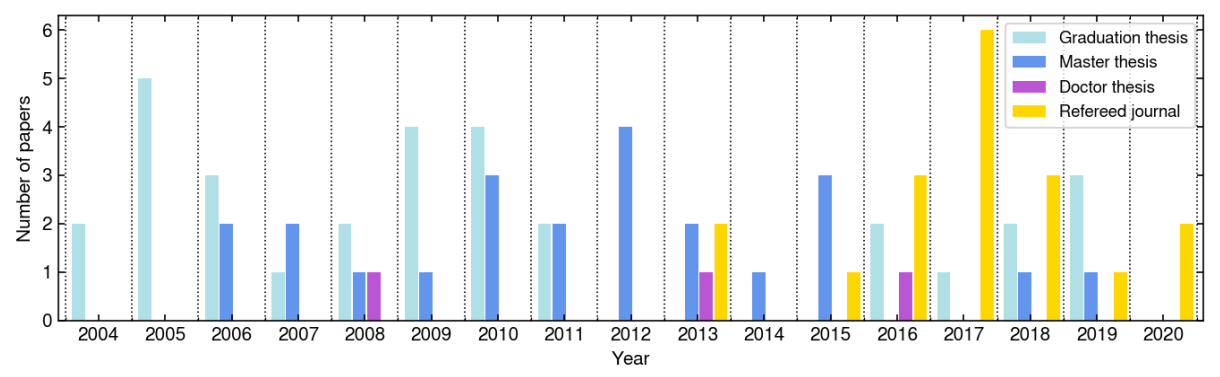

Figure 11. Bar chart indicating numbers of publications related to the 1.85-m telescope project. 


\section{ACKNOWLEDGMENTS}

The 1.85-m telescope project is promoted on a lot of contributions of people engaged in this project, so the authors would like to thank all those people, Takafumi Kojima, Taku Nakajima, Toshihisa Tsutsumi, Ryoko Amari, Kozuki Yuto, Noriaki Arima, Ryosuke Kiridoshi, Takao Matsumoto, Shigeki Osaki, Touga Shiori, Minato Kozu, Yuya Ota, Yoshiharu Kojima, Akio Hashizume, Akihito Minami, Tsubasa Sakaguchi, Hidetoshi Tsuji, Tetsuya Katase, Shimpei Yashima, Masato Kunizane, Masahiro Minowa, Yukimasa Takenaka, Shinpei Yashima, Hirotaka Kurimoto, Atsushi Ezaki, Kazutoshi Maruyama, Hirofumi Okuno, Takashi Nohara, Kiyoko Tsuji, Yoshihide Tokko, Kazuki Toki, Tatsuro Nakayama, and Jun Korogi. We are also grateful to Shigeru Fuji, Akira Mori and Hiroyuki Iwashita, and the entire staff of the Nobeyama Radio Observatory for their useful support. This work was supported by JSPS KAKENHI Grant Numbers JP18H05440, JP15K05025, JP26247026, JP14J12320, JP22244014, and JP15071205, by the Mitsubishi Foundation and the Toray Science Foundation.

\section{REFERENCES}

[1] Dame, T. M. and Thaddeus, P., "A wide-latitude CO survey of molecular clouds in the northern Milky Way.," The Astrophysical Journal 297, 751-765 (Oct. 1985).

[2] Dame, T. M., Ungerechts, H., Cohen, R. S., de Geus, E. J., Grenier, I. A., May, J., Murphy, D. C., Nyman, L. A., and Thaddeus, P., "A Composite CO Survey of the Entire Milky Way," The Astrophysical Journal 322, 706 (Nov 1987).

[3] Dame, T. M., Hartmann, D., and Thaddeus, P., "The Milky Way in Molecular Clouds: A New Complete CO Survey," The Astrophysical Journal 547, 792-813 (Feb 2001).

[4] Fukui, Y. and Yonekura, Y., "Relations Between Star Formation and the Interstellar Medium," in [New Horizons from Multi-Wavelength Sky Surveys], McLean, B. J., Golombek, D. A., Hayes, J. J. E., and Payne, H. E., eds., IAU Symposium 179, 165 (Jan. 1998).

[5] Onishi, T., Mizuno, N., and Fukui, Y., "New View of Molecular Gas Distribution of the Southern Sky: CO Surveys with NANTEN," in [The Dense Interstellar Medium in Galaxies], Pfalzner, S., Kramer, C., Staubmeier, C., and Heithausen, A., eds., 91, 203 (Jan. 2004).

[6] Mizuno, A. and Fukui, Y., [Physical properties of molecular clouds as revealed by NANTEN CO survey: from the galactic center to the galactic warp], vol. 317 of Astronomical Society of the Pacific Conference Series, 59 (2004).

[7] Nishimura, A., Ohama, A., Kimura, K., Tsutsumi, D., Matsue, Y., Yamada, R., Sakamoto, M., Matsunaga, K., Hasegawa, Y., Minami, T., Matsumoto, T., Shiotani, K., Okuda, S., Fujishiro, K., Sakasai, K., Suzuki, M., Saeki, S., Satani, K., Urushihara, K., Kato, C., Kondo, T., Okawa, K., Kurita, D., Inaba, T., Maruyama, S., Koga, M., Noda, K., Kohno, M., Iwamura, H., Hori, Y., Nishikawa, K., Nishioka, T., Bang, J., Sano, H., Enokiya, R., Yoshiike, S., Fujita, S., Hayashi, K., Torii, K., Hayakawa, T., Taniguchi, A., Tsuge, K., Yamane, Y., Hattori, Y., Ohno, T., Ueda, S., Masui, S., Yamasaki, Y., Kondo, H., Suzuki, K., Kobayashi, K., Fujii, Y., Fujii, Y., Minamidani, T., Okuda, T., Yamamoto, H., Tachihara, K., Onishi, T., Mizuno, A., Ogawa, H., and Fukui, Y., "Development of the New Multi-Beam Receiver and Telescope Control System for NASCO," in [Millimeter, Submillimeter, and Far-Infrared Detectors and Instrumentation for Astronomy $X]$, Society of Photo-Optical Instrumentation Engineers (SPIE) Conference Series 11453, 11453-146 (Dec. 2020).

[8] Sakamoto, S., Hayashi, M., Hasegawa, T., Handa, T., and Oka, T., "A Large Area $\mathrm{CO}(\mathrm{J}=2-1)$ Mapping of the Giant Molecular Clouds in Orion," The Astrophysical Journal 425, 641 (Apr 1994).

[9] Sakamoto, S., Hasegawa, T., Hayashi, M., Handa, T., and Oka, T., "An Out-of-Plane CO (J = 2-1) Survey of the Milky Way. I. The Data," The Astrophysical Journal Supplement Series 100, 125 (Sep 1995).

[10] Sorai, K., Hasegawa, T., Booth, R. S., Rubio, M., Morino, J. I., Bronfman, L., Handa, T., Hayashi, M., Nyman, L. A., Oka, T., Sakamoto, S., Seta, M., and Usuda, K. S., "The CO J=2-1/J=1-0 Ratio in the Large Magellanic Cloud," The Astrophysical Journal 551, 794-802 (Apr 2001).

[11] Yoda, T., Handa, T., Kohno, K., Nakajima, T., Kaiden, M., Yonekura, Y., Ogawa, H., Morino, J.-I., and Dobashi, K., "The AMANOGAWA-2SB Galactic Plane SurveyI. Data on the Galactic Equator," Publications of the Astronomical Society of Japan 62, 1277-1289 (Oct. 2010). 
[12] Buckle, J. V., Curtis, E. I., Roberts, J. F., White, G. J., Hatchell, J., Brunt, C., Butner, H. M., Cavanagh, B., Chrysostomou, A., Davis, C. J., Duarte-Cabral, A., Etxaluze, M., di Francesco, J., Friberg, P., Friesen, R., Fuller, G. A., Graves, S., Greaves, J. S., Hogerheijde, M. R., Johnstone, D., Matthews, B., Matthews, H., Nutter, D., Rawlings, J. M. C., Richer, J. S., Sadavoy, S., Simpson, R. J., Tothill, N. F. H., Tsamis, Y. G., Viti, S., Ward-Thompson, D., Wouterloot, J. G. A., and Yates, J., "The JCMT Legacy Survey of the Gould Belt: a first look at Orion B with HARP," Monthly Notices of the Royal Astronomical Society 401, 204-222 (Jan. 2010).

[13] Buckle, J. V., Davis, C. J., di Francesco, J., Graves, S. F., Nutter, D., Richer, J. S., Roberts, J. F., WardThompson, D., White, G. J., Brunt, C., Butner, H. M., Cavanagh, B., Chrysostomou, A., Curtis, E. I., Duarte-Cabral, A., Etxaluze, M., Fich, M., Friberg, P., Friesen, R., Fuller, G. A., Greaves, J. S., Hatchell, J., Hogerheijde, M. R., Johnstone, D., Matthews, B., Matthews, H., Rawlings, J. M. C., Sadavoy, S., Simpson, R. J., Tothill, N. F. H., Tsamis, Y. G., Viti, S., Wouterloot, J. G. A., and Yates, J., "The JCMT Legacy Survey of the Gould Belt: mapping ${ }^{13} \mathrm{CO}$ and $\mathrm{C}^{18} \mathrm{O}$ in Orion A," Monthly Notices of the Royal Astronomical Society 422, 521-541 (May 2012).

[14] Onishi, T., Nishimura, A., Ota, Y., Hashizume, A., Kojima, Y., Minami, A., Tokuda, K., Touga, S., Abe, Y., Kaiden, M., Kimura, K., Muraoka, K., Maezawa, H., Ogawa, H., Dobashi, K., Shimoikura, T., Yonekura, Y., Asayama, S., Handa, T., Nakajima, T., Noguchi, T., and Kuno, N., "A 1.85-m mm-submm Telescope for Large-Scale Molecular Gas Surveys in ${ }^{12} \mathrm{CO},{ }^{13} \mathrm{CO}$, and $\mathrm{C}^{18} \mathrm{O}(\mathrm{J}=2-1), "$ Publications of the Astronomical Society of Japan 65, 78 (Aug 2013).

[15] Sekimoto, Y., Arikawa, Y., Aso, Y., Fujiwara, H., Ikeda, M., Inatani, J., Ito, T., Iwata, M., Kamegai, K., Maezawa, H., Noguchi, T., Ohishi, M., Oka, T., Ozeki, H., Saito, G., Saito, S., Sakai, T., Shi, S.-C., Tatematsu, K., and Yamamoto, S., "The Mt. Fuji submillimeter-wave telescope," in [Radio Telescopes], Butcher, H. R., ed., Society of Photo-Optical Instrumentation Engineers (SPIE) Conference Series 4015, 185-196 (July 2000).

[16] Mizuno, A., Nagahama, T., Morihira, A., Ogawa, H., Mizuno, N., Yonekura, Y., Yamamoto, H., Nakane, H., and Fukui, Y., "Millimeter-Wave Radiometer for the Measurement of Stratospheric ClO Using a Superconductive (SIS) Receiver Installed in the Southern Hemisphere," Journal of Infrared, Millimeter, and Terahertz Waves 23, 981-995 (July 2002).

[17] Nakajima, T., Kaiden, M., Korogi, J., Kimura, K., Yonekura, Y., Ogawa, H., Nishiura, S., Dobashi, K., Handa, T., Kohno, K., Morino, J.-I., Asayama, S., and Noguchi, T., "A New 60-cm Radio Survey Telescope with the Sideband-Separating SIS Receiver for the $200 \mathrm{GHz}$ Band," Publications of the Astronomical Society of Japan 59, 1005 (Oct. 2007).

[18] Benz, A. O., Grigis, P. C., Hungerbühler, V., Meyer, H., Monstein, C., Stuber, B., and Zardet, D., "A broadband FFT spectrometer for radio and millimeter astronomy," Astronomy $\& 3$ Astrophysics 442, 767773 (Nov. 2005).

[19] Asayama, S. and Kamikura, M., "Development of Double-Ridged Wavegide Orthomode Transducer for the 2 MM Band," Journal of Infrared, Millimeter, and Terahertz Waves 30, 573-579 (June 2009).

[20] Kamikura, M., Asayama, S., Satou, N., Shan, W., and Sekimoto, Y., "Development of a Submillimeter Double-Ridged Waveguide Ortho-Mode Transducer (OMT) for the 385-500 GHz Band," Journal of Infrared, Millimeter, and Terahertz Waves 31, 697-707 (June 2010).

[21] Nakajima, T., Skai, T., Kuno, N., and Ogawa, H., "Development of the Measurement System of Image Rejection Ratio for the Sideband-Separating Receiver," Journal of Infrared, Millimeter, and Terahertz Waves 31, 143 (Sept. 2009).

[22] Minamidani, T., Nishimura, A., Miyamoto, Y., Kaneko, H., Iwashita, H., Miyazawa, C., Nishitani, H., Wada, T., Fujii, Y., Takahashi, T., Iizuka, Y., Ogawa, H., Kimura, K., Kozuki, Y., Hasegawa, Y., Matsuo, M., Fujita, S., Ohashi, S., Morokuma-Matsui, K., Maekawa, J., Muraoka, K., Nakajima, T., Umemoto, T., Sorai, K., Nakamura, F., Kuno, N., and Saito, M., [Development of the new multi-beam 100 GHz band SIS receiver FOREST for the Nobeyama 45-m Telescope], vol. 9914 of Society of Photo-Optical Instrumentation Engineers (SPIE) Conference Series, $99141 \mathrm{Z}$ (2016).

[23] Ohyama, H., Nagahama, T., Mizuno, A., Nakane, H., and Ogawa, H., "Observations of stratospheric and mesospheric $\mathrm{O}_{3}$ with a millimeter-wave radiometer at Rikubetsu, Japan," Earth, Planets, and Space 68, 34 (Feb. 2016). 
[24] Asayama, S., Hasegawa, Y., Mizuno, A., Ogawa, H., and Onishi, T., "A Novel Compact Low Loss Waveguide Image Rejection Filter Based on a Backward Coupler with Band Pass Filters for $100 \mathrm{GHz}$ Band," Journal of Infrared 36, 445-454 (May 2015).

[25] Hasegawa, Y., Asayama, S., Harada, R., Tokuda, K., Kimura, K., Ogawa, H., and Onishi, T., "Observational demonstration of a high image rejection SIS mixer receiver using a new waveguide filter at $230 \mathrm{GHz}$," Publications of the Astronomical Society of Japan 69, 91 (Dec. 2017).

[26] Klein, B., Hochgürtel, S., Krämer, I., Bell, A., Meyer, K., and Güsten, R., "High-resolution wide-band fast Fourier transform spectrometers," Astronomy \&3 Astrophysics 542, L3 (Jun 2012).

[27] Hasegawa, Y., Harada, R., Tokuda, K., Kimura, K., Ogawa, H., Onishi, T., Nishimura, A., Han, J., and Inoue, M., "A New Approach to Suppress the Effect of Machining Error for Waveguide Septum Circular Polarizer at $230 \mathrm{GHz}$ Band in Radio Astronomy," Journal of Infrared, Millimeter, and Terahertz Waves 38, 638-652 (May 2017).

[28] Quigley, M., Conley, K., Gerkey, B., Faust, J., Foote, T. B., Leibs, J., Wheeler, R., and Ng, A., "ROS: An open-source robot operating system," in [International Conference on Robotics and Automation], OpenSource Software workshop (2009).

[29] Kondo, H., Matsumoto, takeru, N. A., Ueda, S., Konishi, A., Nakao, y., Nishikawa, K., Nishimoto, s., Fujita, S., Konishi, R., Tsutsumi, T., Takashima, T., Tachihara, K., Onishi, T., and Ogawa, H., "Development of a new software system for radio telescope using Robot Operating System," in [Software and Cyberinfrastructure for Astronomy VI], Society of Photo-Optical Instrumentation Engineers (SPIE) Conference Series 11452, 11452-157 (Dec. 2020).

[30] Shimoikura, T., Dobashi, K., Saito, H., Matsumoto, T., Nakamura, F., Nishimura, A., Kimura, K., Onishi, T., and Ogawa, H., "Molecular Clumps and Infrared Clusters in the S247, S252, and BFS52 Regions," The Astrophysical Journal 768, 72 (May 2013).

[31] Nishimura, A., Tokuda, K., Kimura, K., Muraoka, K., Maezawa, H., Ogawa, H., Dobashi, K., Shimoikura, T., Mizuno, A., Fukui, Y., and Onishi, T., "Revealing the Physical Properties of Molecular Gas in Orion with a Large-scale Survey in $\mathrm{J}=2-1$ Lines of ${ }^{12} \mathrm{CO},{ }^{13} \mathrm{CO}$, and $\mathrm{C}^{18} \mathrm{O}$," The Astrophysical Journal Supplement Series 216, 18 (Jan. 2015).

[32] Da Rio, N., Tan, J. C., Covey, K. R., Cottaar, M., Foster, J. B., Cullen, N. C., Tobin, J. J., Kim, J. S., Meyer, M. R., Nidever, D. L., Stassun, K. G., Chojnowski, S. D., Flaherty, K. M., Majewski, S., Skrutskie, M. F., Zasowski, G., and Pan, K., "IN-SYNC. IV. The Young Stellar Population in the Orion A Molecular Cloud," The Astrophysical Journal 818, 59 (Feb. 2016).

[33] Hacar, A., Alves, J., Forbrich, J., Meingast, S., Kubiak, K., and Großschedl, J., "APOGEE strings: A fossil record of the gas kinematic structure," Astronomy \& Astrophysics 589, A80 (May 2016).

[34] Stutz, A. M. and Gould, A., "Slingshot mechanism in Orion: Kinematic evidence for ejection of protostars by filaments," Astronomy \& Astrophysics 590, A2 (May 2016).

[35] Nakamura, F., Dobashi, K., Shimoikura, T., Tanaka, T., and Onishi, T., "Wide-field ${ }^{12} \mathrm{CO}(\mathrm{J}=2-1)$ and ${ }^{13} \mathrm{CO}(\mathrm{J}=2-1)$ Observations toward the Aquila Rift and Serpens Molecular Cloud Complexes. I. Molecular Clouds and Their Physical Properties," The Astrophysical Journal 837, 154 (Mar. 2017).

[36] Da Rio, N., Tan, J. C., Covey, K. R., Cottaar, M., Foster, J. B., Cullen, N. C., Tobin, J., Kim, J. S., Meyer, M. R., Nidever, D. L., Stassun, K. G., Chojnowski, S. D., Flaherty, K. M., Majewski, S. R., Skrutskie, M. F., Zasowski, G., and Pan, K., "IN-SYNC. V. Stellar Kinematics and Dynamics in the Orion A Molecular Cloud," The Astrophysical Journal 845, 105 (Aug. 2017).

[37] Saajasto, M., Juvela, M., Dobashi, K., Shimoikura, T., Ristorcelli, I., Montillaud, J., Marshall, D. J., Malinen, J., Pelkonen, V. M., Fehér, O., Rivera-Ingraham, A., Toth, L. V., Montier, L., Bernard, J. P., and Onishi, T., "Correlation of gas dynamics and dust in the evolved filament G82.65-02.00," Astronomy 8 Astrophysics 608, A21 (Dec. 2017).

[38] Kounkel, M., Hartmann, L., Mateo, M., and Bailey, John I., I., "Kinematics of the Optically Visible YSOs toward the Orion B Molecular Cloud," The Astrophysical Journal 844, 138 (Aug. 2017). 
[39] Kounkel, M., Covey, K., Suárez, G., Román-Zúñiga, C., Hernandez, J., Stassun, K., Jaehnig, K. O., Feigelson, E. D., Peña Ramírez, K., Roman-Lopes, A., Da Rio, N., Stringfellow, G. S., Kim, J. S., Borissova, J., Fernández-Trincado, J. G., Burgasser, A., García-Hernández, D. A., Zamora, O., Pan, K., and Nitschelm, C., "The APOGEE-2 Survey of the Orion Star-forming Complex. II. Six-dimensional Structure," The Astronomical Journal 156, 84 (Sept. 2018).

[40] Santos-Silva, T., Gregorio-Hetem, J., Montmerle, T., Fernandes, B., and Stelzer, B., "Star formation history of Canis Major OB1. II. A bimodal X-ray population revealed by XMM-Newton," Astronomy E Astrophysics 609, A127 (Feb. 2018).

[41] Fernandes, B., Montmerle, T., Santos-Silva, T., and Gregorio-Hetem, J., "Runaways and shells around the CMa OB1 association," Astronomy \& Astrophysics 628, A44 (Aug. 2019).

[42] Peñaloza, C. H., Clark, P. C., Glover, S. C. O., Shetty, R., and Klessen, R. S., "Using CO line ratios to trace the physical properties of molecular clouds," Monthly Notices of the Royal Astronomical Society 465, 2277-2285 (Feb. 2017).

[43] Masui, S., Minami, T., Okawa, M., Yamasaki, Y., Yokoyama, K., Ueda, S., Hasegawa, Y., Onishi, T., Ogawa, H., Kojima, T., and Gonzalez, A., "Development of a wideband waveguide diplexer forsimultaneous observation at 210-375 GHz," in [Millimeter, Submillimeter, and Far-Infrared Detectors and Instrumentation for Astronomy X], Society of Photo-Optical Instrumentation Engineers (SPIE) Conference Series 11453, 11453-165 (Dec. 2020).

[44] Yamasaki, Y., Masui, S., Okawa, M., Yokoyama, K., Minami, T., Ueda, S., Hasegawa, Y., Nishimura, A., Ogawa, H., Onishi, Toshikazu, O. N., Kimura, K., Gonzalez, A., Kojima, T., Kaneko, K., and Sakai, R., "Optical design of the 1.85-m mm-submm telescope in $210-375 \mathrm{GHz}$ band," in [Millimeter, Submillimeter, and Far-Infrared Detectors and Instrumentation for Astronomy X], Society of Photo-Optical Instrumentation Engineers (SPIE) Conference Series 11453, 11453-172 (Dec. 2020). 